\title{
An Optimized Type-2 Self-Organizing Fuzzy Logic Controller Applied in Anesthesia for Propofol Dosing to Regulate BIS
}

Wei, Zi-Xiao; Doctor, Faiyaz; Liu, Yan-Xin; Fan, Shou-Zen; Shieh, Jiann-Shing

Published in:

IEEE Transactions on Fuzzy Systems

Link to article, DOI:

10.1109/tfuzz.2020.2969384

Publication date:

2020

Document Version

Peer reviewed version

Link back to DTU Orbit

Citation (APA):

Wei, Z-X., Doctor, F., Liu, Y-X., Fan, S-Z., \& Shieh, J-S. (2020). An Optimized Type-2 Self-Organizing Fuzzy

Logic Controller Applied in Anesthesia for Propofol Dosing to Regulate BIS. IEEE Transactions on Fuzzy

Systems, 28(6), 1062-1072. https://doi.org/10.1109/tfuzz.2020.2969384

\section{General rights}

Copyright and moral rights for the publications made accessible in the public portal are retained by the authors and/or other copyright owners and it is a condition of accessing publications that users recognise and abide by the legal requirements associated with these rights.

- Users may download and print one copy of any publication from the public portal for the purpose of private study or research.

- You may not further distribute the material or use it for any profit-making activity or commercial gain

- You may freely distribute the URL identifying the publication in the public portal 


\title{
An Optimized Type-2 Self-Organizing Fuzzy Logic Controller Applied in Anesthesia for Propofol Dosing to Regulate BIS
}

\author{
Zi-Xiao Wei, Faiyaz Doctor, Member, IEEE, Yan-Xin Liu, Shou-Zen Fan, Jiann-Shing Shieh
}

\begin{abstract}
During general anesthesia, anesthesiologists who provide anesthetic dosage traditionally play a fundamental role to regulate Bispectral Index (BIS). However, in this paper, an optimized type-2 Self-Organizing Fuzzy Logic Controller (SOFLC) is designed for a Target Controlled Infusion (TCI) pump related to propofol dosing guided by BIS, to realize automatic control of general anesthesia. The type-2 SOFLC combines a type2 fuzzy logic controller with a self-organizing (SO) mechanism to facilitate online training while able to contend with operational uncertainties. A novel data driven Surrogate Model (SM) and Genetic Programming (GP) based strategy is introduced for optimizing the type-2 SOFLC parameters offline to handle interpatient variability. A pharmacological model is built for simulation in which different optimization strategies are tested and compared. Simulation results are presented to demonstrate the applicability of our approach and show that the proposed optimization strategy can achieve better control performance in terms of steady state error and robustness.
\end{abstract}

Index Terms - anesthesia; type-2 fuzzy controller; optimization; surrogate model; genetic programming

\section{INTRODUCTION}

G ENERAL anesthesia is a vital component of modern medicine involving medically induced unconsciousness, systemic analgesia, reflex inhibition and muscle relaxation. During the general anesthesia procedure, achieving unconsciousness is a potentially risky and protean process, which demands accurately monitoring the Depth of Anesthesia (DoA) and providing reasonable control of anesthetic dosage to maintain a safe anesthetized state for the patient.

Electroencephalography (EEG) is the most widely used

Manuscript received June 28, 2019; revised November 11, 2019; accepted January 13, 2019. (Corresponding author: Faiyaz Doctor, Co-corresponding author: Jiann-Shing Shieh)

Zi-Xiao Wei is with the Department of Mechanical Engineering, National University of Singapore, 119077, Singapore. (e-mail: e0452857@u.nus.edu).

Faiyaz Doctor is with the Centre for Computational Intelligence, School of Computer Science and Electronic Engineering, University of Essex, Colchester CO4 3SQ, U.K. (e-mail: fdocto@essex.ac.uk).

Yan-Xin Liu is with the Department of Physics, Technical University of Denmark, Kongens Lyngby, 29042, Denmark (e-mail: yanxinliu@dtu.dk).

Shou-Zen Fan is with the Department of Anesthesiology, National Taiwan University Hospital, Taipei, 100, Taiwan (e-mail: shouzen@ntuh.gov.tw).

Jiann-Shing Shieh is with the Department of Mechanical Engineering, Yuan Ze University, Chung-Li, Taoyuan, 320, Taiwan (e-mail: jsshieh@saturn.yzu.edu.tw) method clinically related to the indirect DoA monitoring [1]. Anesthesia delivery is traditionally controlled by experienced anesthesiologists manually guided by Bispectral Index (BIS), which can be dissected from spectral analysis of the raw EEG using fast Fourier techniques. Nevertheless, maintaining DoA is a cognitively intensive process especially during long and complicated multi-stage surgical procedures. Errors in infusion rates and dosage can impact on the patient's post-operative recovery and safety.

Automatic controllers have been applied in many circumstances to reduce human errors and alleviate the heavy burden on medical staff. Over the past two decades, the development of Target Controlled Infusion (TCI) pumps for propofol infusion based on pharmacological data of patients and Computer Assisted Continuous Infusion (CACI) has proved its superiority to manually controlled syringe pumps related to induction and maintenance of anesthesia [2]. The human body is a highly nonlinear system with high level of inherent uncertainties leading to challenges in achieving automated processes. TCI has been successfully and commercially applied in clinical general anesthesia. However, it is an open loop control system and unable to handle these uncertainties or adaptability to different operating conditions [3]. Recent researchers have focused more on closed loop control infusion of anesthesia $[4,5]$ including propofol dosing to regulate BIS [6,7]. Different metabolism rates between patients and disturbances in the surgical environment such as blood loss, however, lead to considerable data loss and inaccuracies of traditional PID controllers.

Hierarchical intelligent controllers with feedback strategies have been implemented for dealing with these nonlinear biomedical systems. Here fuzzy logic controllers (FLC) provide a powerful methodology for handling data uncertainties [8,9]. Recently, researchers have used type-2 fuzzy sets to provide robustness for handling short- and long-term uncertainties in anesthesia control [10]. Type-2 FLCs based on type-2 fuzzy sets have been shown to be able to handle uncertain ranges of parameters arising from noise and external factors affecting the environment [11]. This is due to type-2 fuzzy sets having greater design degrees of freedoms, which can handle higher orders of real-world uncertainties to produce potentially more accurate and stable control systems [12-17].

Nevertheless, type-2 fuzzy logic systems are not always able to achieve an optimal performance while coping with multiple control effects such as intra- and inter-patient variability, pharmacokinetic and pharmacodynamic variability 
associated with biomedical processes. Firstly, the basic type of FLC, be it type-1 or type-2, is a fixed system and does not have any adaptability for handling changing operating conditions as the monitored patient's response varies during surgery. Secondly, determining the optimum structure of the fuzzy logic system, the design of rule-base, type- 2 fuzzy sets and scaling factors is challenging. This has led to recent efforts focused on applying adaptation mechanisms and machine learning to enhance FLC design. One adaptive FLC which had been successfully applied to anesthesia control is the Self-Organizing Fuzzy Logic Controller (SOFLC), which adopts a SelfOrganizing (SO) mechanism enabling online adaptation of FLCs [18]. The SO mechanism is a top-down, spontaneous system which has been developed to enhance the capability of FLCs to deal with dynamic changes caused by external stimuli or drug effects during anesthesia. Based on our previous research, general and Interval Type-2 (IT2) SOFLCs were successfully applied to anesthesia control for maintaining acceptable DoA $[10,19]$ where they were shown to outperform type-1 based systems. Here clinical patient data was used in specifying the type-2 Membership Functions (MFs) to capture intra and inter-patient variability. However, the fuzzy sets and other control parameters of the FLC could be suboptimal with respect to different combinations of surgical conditions and patient specific physiological characteristics impacting on its control performance. There is subsequently a need to efficiently optimize the parameters of the type-2 SOFLC for enhancing the online adaptive control of drug infusion in maintaining DoA.

\section{RELATED LITERATURE}

Previous related research has utilized different algorithms to adapt and optimize the parameters of type- 1 and type-2 FLCs for anesthesia control. Several studies have focused on simulating the control of drug infusion rates for regulation of blood pressure and muscle relaxation as part of a multivariable anesthesia system. In [20] a direct adaptive IT2-FLC has been applied where the approach uses a predefined rule-base and adjusts the centers of the output MFs of fired rules in response to deviations from the desired control response. In [21] an approach based on IT2 Fuzzy Neural Network (IT2FNN) controller is evaluated where the components of an IT2 FLC are defined as layers of the IT2FNN and a backpropagation algorithm is used to tune the centers and spreads of the input IT2 MFs. More recently a Genetic Algorithm (GA) is used in [22] to optimize the centers of the output MFs of an IT2 FLC for regulating DoA. These approaches, however, either randomly select or manually add inter and intra-variability for specific surgical scenarios rather than this being derived using real patient data. In [16] authors have applied GAs to optimise the parameters of type- 1 and type-2 PIDs controllers for regulating BIS. Here patient data is used to define the parameter of the pharmacological anesthesia model and a GA is used to optimize the PID gains, scaling factors and input and output IT2 MF parameters. Similarly, in [23] an approach using a model predictive controller based on an adaptive type- 1 fuzzy model is used to regulate BIS. Patient data is again used to define a generic patient model in the form of fixed fuzzy control rules. A GA is applied online to compute the input fuzzy set parameters so that drug dosage can be adapted to patient specific characteristics [24].

Unlike the aforementioned techniques, SOFLC uses an online self-adaptive mechanism that is based on the modification of its fuzzy control rules. Our previous work has explored the use of GAs to optimize the SOFLC interval type2 fuzzy sets and scaling factors [25]. However, GAs involve running simulations for evaluating the fitness of every individual (candidate SOFLC) in a generated population over multiple generations which has proven to be a highly timeconsuming and computationally expensive process. This is due to the complicated iteration procedure and large control parameter space representing each candidate controller. Motivated by the desire to solve these problems, an efficient optimization algorithm using Surrogate Models (SM), have been developed in reference to multidisciplinary design optimization [26], and used to optimize the structure of fuzzy logic systems [27]. The algorithm adopts a response surface methodology to obtain an optimal response model, which significantly reduces the simulation time and complexity without influencing quality of the offspring while handling computationally expensive problems.

However, differences between the patients' physiological characteristics such as age, weight and height, may still result in unsatisfactory control performance. The optimal SM based solution of a single SOFLC optimization case may not be the optimal solution in consideration of different patient specific characteristics or might even be unfeasible. This is especially true for patients who are over or underweight, where the control performance tends to be unsatisfactory. Running an optimization process every time before the surgery for a specific patient is also unrealistic. Genetic Programming (GP) provides a methodology which can be used for automatically evolving interpretable symbolic regression models for discovering nonlinear functional relationships in data $[28,29]$ without requiring any prior specification of the form or structure of the solution in advance [30]. This enables GP to build symbolic regression, which can simultaneously search for both the complex associations between predictors and outcome and the optimal model structure.

GP is therefore considered as a powerful technique for program configuration and has been previously applied in [31, 32] for optimizing rules of FLCs. In our work it is used to alleviate the problem of requiring to run online optimization processes to configure the SOFLC to patient specific characteristics. GP evolves tree structured program which can be used to replace the fixed constants of SM optimized type-2 fuzzy sets and scaling factors. The evolved programs can find the relationship between the patient information and parameters of the type-2 SOFLC to provide appropriate parameters of type2 fuzzy sets and scaling factors for each anesthesia case.

This paper proposes a holistic data-driven optimized fuzzy adaptive control scheme for controlling propofol dosing to regulate BIS as an indicator for DoA. This novel approach combines offline SM and GP based optimization of type-2 MFs and scaling factors with the online adaptation of the fuzzy control rules of the type-2 SOFLC. To the authors' knowledge, there have been no previously conducted studies which have combined SM and GP for optimizing and flexibly selecting 


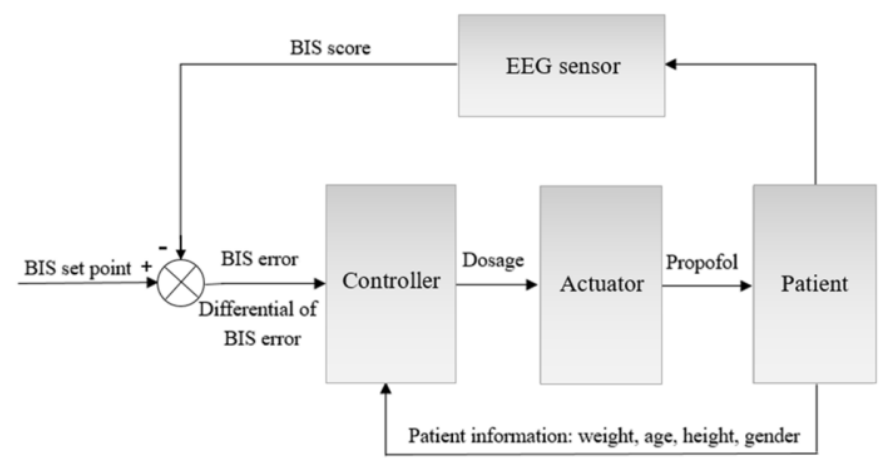

Fig. 1 Diagram of the multivariable anesthesia system

parameters of adaptive type-2 FLCs applied to anesthesia control. The contribution of this paper is therefore: 1) the application of SM to optimize the input IT2 MF parameter, and scaling factors of type-2 SOFLCs; 2) Using GP to determine the best set of optimized SOFLC parameters for patient specific characteristics under simulated surgical scenarios. We compare and evaluate the control performance of the proposed system with type-2 SOFLCs optimized by GA and SM as well as an equivalent type-1 system.

The rest of this paper is organized as follows: in section III, the pharmacological model of propofol used in our simulation is introduced; in section IV, the IT2 SOFLC is explained; in section $\mathrm{V}$, approaches for optimizing the type-2 SOFLCs are described in detail; in section VI, the experiments and results are presented and discussed, Finally, section VII presents conclusions and future works.

\section{PHARMACOLOGICAL MODEL OF PROPOFOL}

As a versatile anesthetic agent, propofol is an intravenous short-acting medication used for induction and maintenance of general anesthesia and hence widely used for computer controlled anesthesia $[1,6]$. Moreover, BIS values ranging from 0 to 100 , are used by clinicians to interpret DoA. Awake patients commonly show a BIS value greater than 90. BIS scores between 40 and 60 are considered reasonable during adequate levels of general anesthesia [33]. In this paper, a nonstatic anesthetic model is applied as a reference model for the proposed type-2 SOFLC to realize closed loop control of the infusion rate of propofol for regulating DoA as described in Fig. 1. The hierarchical system consists of a type-2 FLC (a), a SO mechanism (b) to facilitate online tuning of the type-2 FLC control rules, and the combination of SM and GP to facilitate offline learning (c) and define the type-2 FLC system structure. The patient information is firstly sent to the controller to determine controller parameters. Then the controller predicts appropriate dosage for the actuator to deliver propofol based on the BIS set point, and the BIS error. The differential of BIS error is calculated and fed back to the controller after the EEG sensor provides a BIS score from the patient. In our surgical simulation, a propofol pharmacological model of the patient is applied. According to previous studies [34], the pharmacological model of propofol is usually divided into two classes to clarify the interaction and mechanism of the drug and the patient. The pharmacokinetics (PK) model is related to the rules governing the concentration of the drug in the body which is affected by the absorption, distribution, metabolism and excretion of drugs in the body.

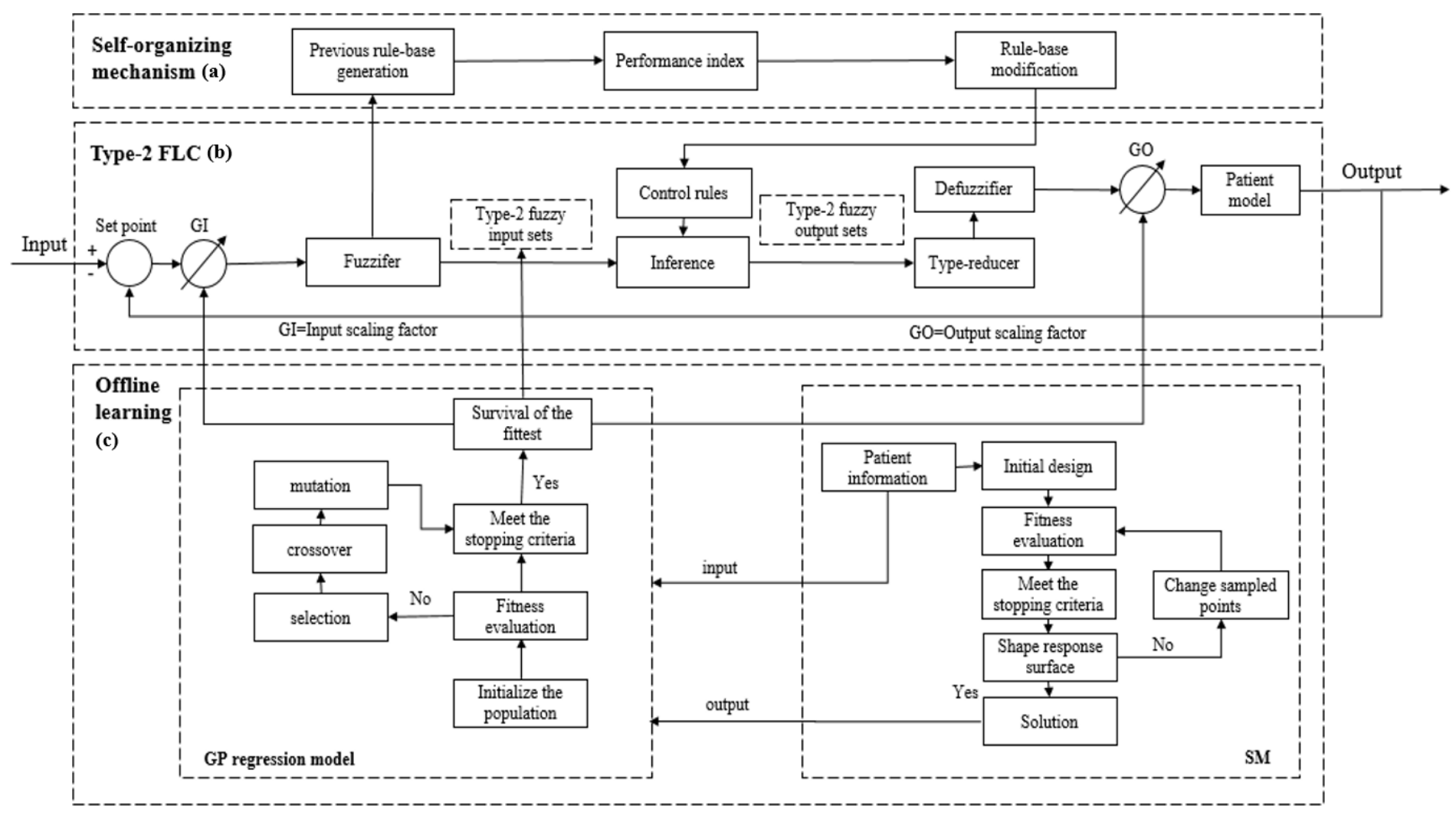

Fig. 2 Schematic diagram of the proposed type-2 SOFLC: (a) Self-organizing mechanism (b) Type-2 FLC (c) Offline learning 
The pharmacodynamics (PD) model is meant to study the effect of drugs on the body including drug action, mechanism of action and clinical application. Both models together represent the patient model as illustrated as a block in Fig. 1 and Fig. 2. The mathematical formalism of both these models are described in more detail in the sections below.

\section{A. Pharmacokinetics model}

Based on previous studies [34], the free plasma propofol concentration was described using a three-compartment model:

$$
\begin{gathered}
V_{p} \frac{d C_{P}}{d t}=-\left(C L+Q_{1}+Q_{2}\right) C_{P}+Q_{1} C_{T 1}+Q_{1} C_{T 2}+R \\
V_{T 1} \frac{d C_{T 1}}{d t}=Q_{1} C_{P}-Q_{1} C_{T 1} \\
V_{T 2} \frac{d C_{T 2}}{d t}=Q_{2} C_{P}-Q_{2} C_{T 2}
\end{gathered}
$$

where $C_{P}, C_{T 1}$ and $C_{T 2}$ denote concentrations of propofol in the three compartments. $V_{p}, V_{T 1}$ and $V_{T 2}$ denote volumes of distribution of the three compartments and $C L$ denotes metabolic clearance of propofol. $Q_{1}$ and $Q_{2}$ denote the intercompartmental clearances. $R$ denotes the infusion rate.

\section{B. Pharmacodynamics model}

Based on previous studies [35], the BIS value can be calculated corresponding to the effect-site concentrations through a sigmoidal $E_{\max }$ model:

$$
B I S(t)=B I S_{0}\left(1-E_{\max } \frac{C_{e}^{\gamma}}{E C_{50}^{\gamma}+C_{e}^{\gamma}}\right)
$$

where $B I S_{0}$ denotes the baseline of BIS score, which is conventionally assigned a value of $100 . E_{\max }$ is a constant fixed to 1 , involving maximal effect. $E C_{50}$ is a constant fixed to 7.5, denoting the concentration for a $50 \%$ decrease in BIS score. $\gamma$ is a constant fixed to 3 , determining the steepness of the curve. $C_{e}$ is a variable representing the effect compartment concentration parameter which can be calculated as follows:

$$
\frac{d C_{e}}{d t}=k_{e 0}\left(C_{P}-C_{e}\right)
$$

where $k_{e 0}$ represents biophase distribution rate constant fixed to 0.456 , and $C_{P}$ is a variable representing the central compartment's concentration of propofol.

\section{TYPE-2 SOFLC}

Our proposed type-2 SOFLC for anesthesia control consists of a type-2 FLC and a SO mechanism, which is a closed loop controller with a hierarchical structure as presented in Fig. 2. Both these components of type-2 SOFLC will be described in the sections as follows.

\section{A. Interval type-2 FLC}

The type-2 FLC consists of a fuzzifier, inference engine, rule-base, type-reducer, and a defuzzifier as proposed by $\mathrm{J}$. M. Mendel in 1999 [36]. The type-2 FLC is characterized by a fuzzy MF, where the membership value (or membership grade) for each element of the set is itself a fuzzy set in $[0,1]$. This allows us to capture and handle uncertainties about the degree of membership of an element in the fuzzy set [37]. The fuzzy membership grades reflect parameter variabilities observed in data which are captured in the Footprint of Uncertainty (FOU)

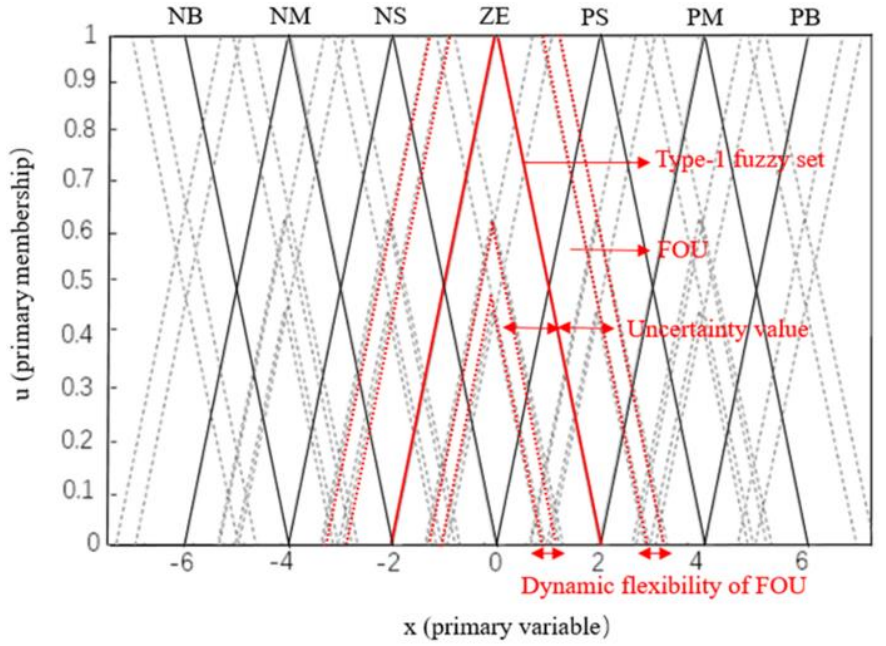

Fig. 3 Interval type-2 fuzzy set employed in the proposed type-2 SOFLC

of the type-2 fuzzy set. This gives type-2 FLC parameters more design degrees of freedom to represent and manage uncertain data driven problems. Hence, a type-2 FLC has been shown to perform better in handling nonlinear biomedical control processes such as anesthesia regulation. According to comparisons with different kinds of type-2 FLC, J.M. Mendel proposed an Interval Type-2 (IT2) FLC whose secondary MF is a fixed interval. IT2 FLC is considered as an efficient controller to solve real world problems [38] such as anesthesia control [10]. Several application areas have successfully applied IT2 fuzzy sets to reduce the computational burden owing to its simplicity compared with general type-2 fuzzy sets. IT2 FLCs also provide a proven basis for introducing and evaluating hybrid evolutionary optimization approaches for tuning the fuzzy system's parameters to enhance its performance [23, 24]. In this paper, we apply IT2 fuzzy sets for representing the inputs and output of the SOFLC following previous studies [39-41]. As described in Fig. 2 (b), the proposed type-2 FLC is a twoinputs single-output system based on singleton fuzzification. The inputs of the type-2 FLC are the feedback error and differential error of BIS, which will be transformed into a normalized universe of discourse for use in the controller using input scaling factors depicted as GI in Fig. 2 (b). The fuzzy rule of the 2 inputs and 1 output system can be described as "IFTHEN" rules of the form:

$$
\text { IF } x_{1} \text { is } \tilde{F}_{1}^{l} \text { and } x_{2} \text { is } \tilde{F}_{2}^{l} \text {, THEN } y \text { is } \tilde{G}^{l} \text {. }
$$

where $x_{1}$ and $x_{2}$ are inputs, $\widetilde{F}$ is the input fuzzy set, $y$ is the output, $\tilde{G}$ is output fuzzy set and $l$ denotes the activated rule.

As shown in Fig. 3, seven linguistic values are used to define the two inputs as follows: negative big (NB), negative medium $(\mathrm{NM})$, negative small (NS), zero (ZE), positive small (PS), positive medium (PM), and positive big (PB). Here, the type-2 FOUs of the sets $\mathrm{NB}$ and $\mathrm{PB}$ are constructed based on parallelogram MFs extending the triangular type-1 fuzzy sets previously used [37, 42]. The FOU of the IT2 fuzzy set is bounded by both a trapezoidal Upper MF and a triangular Lower MF. For example, the IT2 fuzzy set ZE is constructed by adding a symmetric uncertainty value to a type-1 MF as Fig. 3 shows. The uncertainty value and the dynamic flexibility of FOUs (as shown by the dashed lines in Fig 3) in handling 
changing and inter subject specific variabilities, can be achieved using effective optimization schemes by combining SM and GP as our proposed approach illustrates in section IV. The inference engine uses rules activated according to input type-2 fuzzy sets to infer the output type-2 fuzzy sets by applying union operations and type- 2 intersection based on tconorm and t-norm operations respectively, which is a simplified and efficient way to compute the input and antecedent operations of the IT2 fuzzy sets [43]. The typereducer then combines the output type- 2 fuzzy sets to form a type-1 fuzzy set which is known as the type reduced set [44]. In this study we implement the enhanced iterative algorithm with stopping condition method proposed by $\mathrm{Wu}$ et al. [45] as the type-reducer. Four linguistic values are used to define the output propofol infusion rate as follows: ZE, PS, PM, and PB. The fuzzy sets for the outputs are simplified to intervals with center-of-sets type-reduction method, which are based on the centroids of the IT2 fuzzy sets [37, 42, 43]. The simplified output fuzzy sets are four sub-intervals, which are the equal divisions of the interval $[0,1]$, and these remain fixed in the optimization process in order to reduce complexity. The controller's crisp output is also transformed using the output scaling factor depicted as GO corresponding to the propofol infusion rate as shown in Fig. 2 (b). Further information on the IT2 fuzzy set can be found in $[37,38]$.

\section{B. Self-organizing mechanism}

As there are 7 linguistic values of the input fuzzy sets to define 2 input variables, the max number of possible rules is 49 .

TABLE I

INITIAL RULE-BASE DESIGNED BASED ON ANESTHESIOLOGIST'S KNOWLEDGE

\begin{tabular}{|c|c|c|c|c|c|c|c|}
\hline \multirow{2}{*}{ Error of BIS } & \multicolumn{7}{|c|}{ Differential error of BIS } \\
\cline { 2 - 9 } & NB & NM & NS & ZE & PS & PM & PB \\
\hline NB & ZE & & ZE & & ZE & & ZE \\
\hline NM & & ZE & & ZE & & ZE & \\
\hline NS & ZE & & ZE & & ZE & & PS \\
\hline ZE & & ZE & & PS & & ZE & \\
\hline PS & ZE & & ZE & & PS & & PM \\
\hline PM & & PM & & PM & & PM & \\
\hline PB & PM & & PM & & PB & & PB \\
\hline
\end{tabular}

TABLE II

LINGUISTIC PERFORMANCE INDEX TABLE

\begin{tabular}{|c|c|c|c|c|c|c|c|}
\hline \multirow{2}{*}{ Error } & \multicolumn{7}{|c|}{ Change in error } \\
\cline { 2 - 8 } & NB & NM & NS & ZE & PS & PM & PB \\
\hline NB & NB & NB & NB & NM & NM & NS & ZE \\
\hline NM & NB & NB & NM & NM & NS & ZE & NS \\
\hline NS & NB & NB & NS & NS & ZE & PS & PM \\
\hline ZE & NB & NM & ZE & ZE & PS & PM & PB \\
\hline PS & NM & NS & ZE & PS & PS & PB & PB \\
\hline PM & NS & ZE & PS & PM & PM & PB & PB \\
\hline PB & ZE & PS & PM & PM & PB & PB & PB \\
\hline
\end{tabular}

The rule base applies a SO mechanism to facilitate online tuning, which means modifying the controller during its running process to enhance the capability and adaptability of FLCs to deal with dynamic changes caused by external stimuli or drug effects in anesthesia. The SO mechanism incorporates three function blocks: the previous rule-base generation, performance index and rule-base modification as shown in Fig. 2 (a).

The initial rule-base was generated from expert experience (i.e., anesthesiologist knowledge) as shown in Table I which initially has 25 rules to start with. The performance index evaluates the deviation from the desired response to the feedback from patient model and calculates the required changes in the output of the controller. This is based on assigning a reward value $P$ to the individual control actions that contributed more to the present performance. The reward value $P$ is generated from a fuzzy algorithm to define the desired performance in the form of fuzzy linguistic values, which are shown in Table II, derived from previous research work in [40].

The generation and modification of the existing control rules will be achieved by the rule-base modification block. Given $T$ is a sampling time step and $n$ is the number of sampling steps, suppose at time $n T$, the process has a time-lag of $m$ samples, the inputs of the system are $e(n T)$ and $c e(n T)$, the output of the controller is $u(n T)$. Here $e, c e, u$ represent error, change error and controller output respectively.

For each control performance, the singleton fuzzy inputs of $e(n T)$ and $c e(n T)$ will stimulate two fuzzy subsets and help to define the reward value $P(n T)$ issued by performance index defined in Table II. Then the control action $u(n T-m T)$ contributing most to the process performance corresponding to $e(n T-m T)$ and $c e(n T-m T)$ should be $u(n T-m T)+P(n T)$ instead of $u(n T-m T)$. A fuzzy operator $\mathrm{F}\{\}$, is applied for fuzzifying the parameters into linguistic value as follows:

$$
\begin{gathered}
E(n T-m T)=F\{e(n T-m T)\} \\
C E(n T-m T)=F\{c e(n T-m T)\} \\
U(n T-m T)=-F\{u(n T-m T)\} \\
V(n T-m T)=-F\{u(n T-m T)+P(n T)\}
\end{gathered}
$$

then the previous implication:

$$
E(n T-m T) \rightarrow C E(n T-m T) \rightarrow U(n T-m T)
$$

will be replaced by the new one:

$$
E(n T-m T) \rightarrow C E(n T-m T) \rightarrow V(n T-m T)
$$

As mentioned, $P$ is the reward value issues by the performance index and $E, C E, U$ represent linguistic error, linguistic change error and linguistic controller output respectively depicted in Table II. $V$ is the modified rule.

After the rule modification procedure, a new rule will be generated by the method of logic examination [46] from the input and output data of the controller at each sampling step. If the new generated rule does not exist in the rule-base, it will be added. Otherwise, if it is already present, it will be ignored. Further information on the SO mechanism can be found in [47].

\section{OFFLINE TRAINING}

In this section, the SM-GP based optimization of type-2 SOFLC through altering the width of FOU for type-2 fuzzy sets and adjusting the two input and output scaling factors is proposed. The schematic diagram in Fig. 2 (c) shows the interaction of combined SM and GP optimization processes and 
type-2 SOFLC. This optimization process is offline regarding to the controller structure configuration. We have primarily used the information of 40 patients to perform the SM optimization to achieve the optimized parameters. GP is applied subsequently to find the relationship between the patient information and optimized parameters. The GP inspired from the mechanisms of natural selection and genetics for automatically generating programs based on stochastic search techniques, will help define the type-2 SOFLC structure.

\section{A. Surrogate Model}

Surrogate modeling is an engineering method extensively used in the design community, and recently has been applied in many other areas such as computationally expensive experiments, function evaluations or optimization procedures to approximate complex simulation-based models [48].

Data on average height, weight and age was acquired for 40 patients from National Taiwan University Hospital. For each patient, we ran SM optimization to find the optimal controller parameters including the FOU widths for input type-2 fuzzy sets and the two input and single output scaling factors. The objective function to be minimized here was defined as the steady state error from the training simulation runs of the type2 SOFLC. This has the effect of reducing the deviation of the control output from the set point and is used to produce a smooth control response and reduce fluctuation of BIS based on the propofol infusion rate.

SM optimization commonly obeys the following steps as described in Fig. 2 (c). Firstly, an initial point set is randomly created and function evaluations are performed on the selected points. The method then shapes a response surface corresponding to the data generated and uses it to predict the fitness of the objective function at unsampled points within the variable domain. This process helps to select points to perform the next evaluation. The approach continually keeps performing the evaluation on the set of selected points and uses the fittest point set to update the model until the stopping criterion has been met. Typically, the stopping criteria is a maximum number of allowed function evaluations, which we define as 200.

We chose a mixture of cubic radial basis function interpolant and kriging model with Gaussian correlation function for specifying the response surface. Candidate Point Sampling Strategy (CAND) is used as the sampling strategy and the Symmetric Latin Hypercube Sampling Strategy (SLHD) is used as the experimental design strategy. In addition, we define the number of points in the initial experimental design to be 10 , which has been shown to performs well in 4-dimensional problems [48].

\section{B. Genetic Programming}

GP is a branch of evolutionary artificial intelligence introducing the methodologies of Automatically Defined Functions (ADF), which relevels specific abilities to develop relationships between specific system inputs and outputs and has been applied in several areas [49]. GP based regression can produce relatively simple predictive models through the evolutionary discovery and optimization of unknown coefficients for determining nonlinear system input/output relationship [29]. Unlike other machine learning techniques such as Artificial Neural Networks and Support Vector
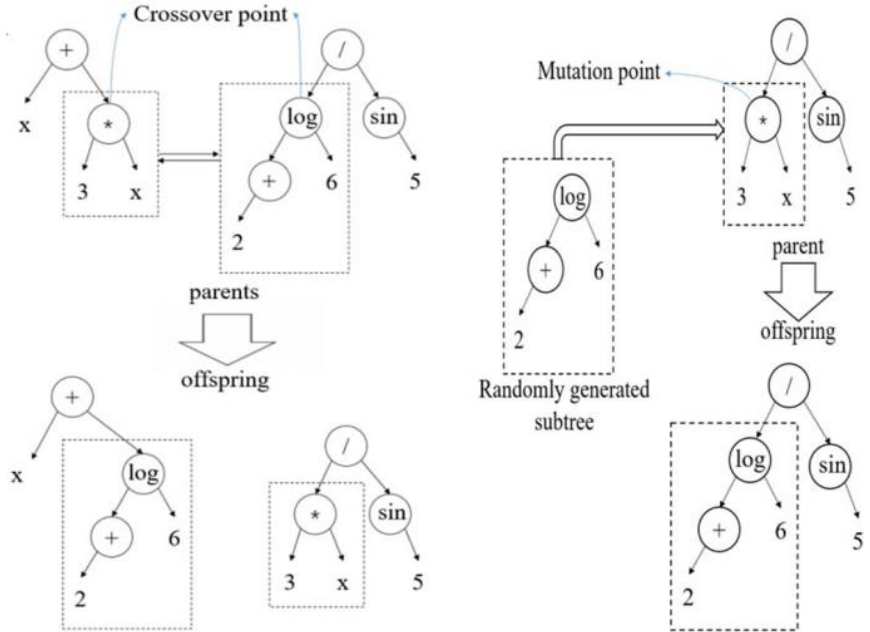

(a)

(b)

Fig. 4 Diagrams of (a) subtree crossover and (b) subtree mutation

Machines. GP can simultaneously determine interpretable expressions to explain this functional relationship [29, 50, 51] and achieve reasonable generalizing behavior as compared to other approaches. As the patient characteristics impact on the pharmacokinetics and pharmacodynamics model of propofol, GP is an efficient method to find the relationship between patient information and effects of different parameter settings.

GP develops its structure in the adaptive evolution of programs where individuals are usually expressed as syntax trees rather than strings (as is the case with GAs). As described in Fig. 2 (c), during the GP running process, the initial population is generated randomly. Typically, the structures of individuals are configured with the aid of branches. GP implements the following genetic operators: Fitness Evaluation, Selection, Crossover and Mutation. Individuals are selected through these genetic operators for producing the next generation of offspring. The process of measuring fitness is to sum the absolute error. GP continues generating new population and performing fitness evaluation until a stopping criteria is met. The implementation of these operators makes GP significantly different from other evolutionary algorithms [30]. In Fig. 4, examples of crossover and mutation operations are shown. The subtree crossover operator randomly selects a crossover point and creates new offspring by exchanging the branches rooted with the parent trees. The subtree mutation operator selects a mutation point and replaces the branches rooted with a randomly generated subtree [30]. Simplification and encapsulation are two other secondary operators to simplify the redundant symbols of complex trees and encapsulate the most frequent subtree into a user-defined function. The population size, generation and reproduction rate in our work are defined as 1000, 100 and 0.1 respectively. The possibility of crossover and mutation to generate new individuals is defined as 0.5 and 0.3 respectively. Tournament selection is applied for generating a mating pool, which is robust in noisy environments.

To apply GP, we used the data on the height, weight and age acquired from the 40 patients to perform patient specific SM optimizations of the type-2 SOFLC parameters. This resulted in 4 datasets corresponding to the FOU for input type-2 fuzzy sets, 


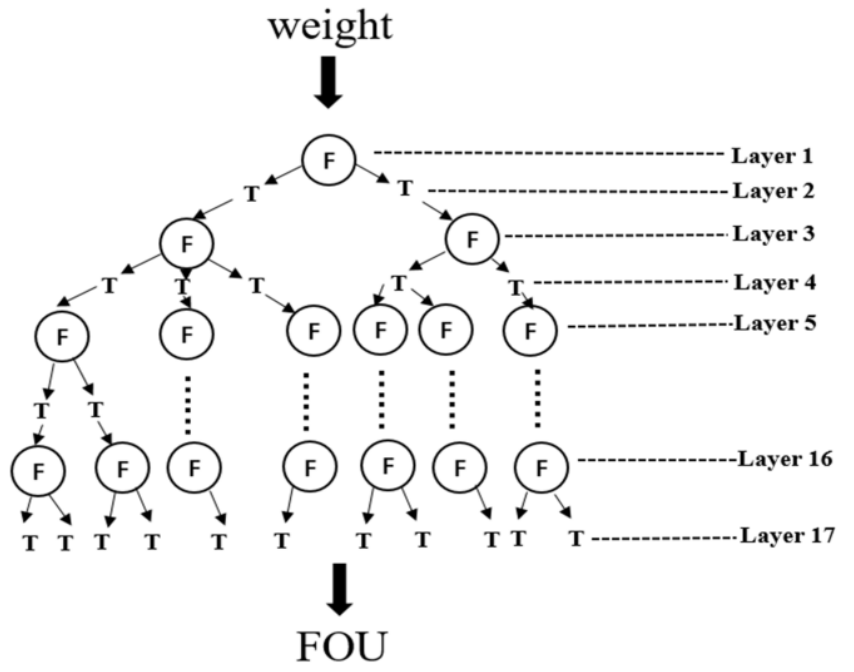

Fig. 5 An example of a GP tree

two input scaling factors and one output scaling factor, which are defined as the output of GP. We define the input set of the GP to be the weights of the 40 patients as weight is the most significant factor observed to impact anesthesia regulation. The GP can then be used to build regression models, which will replace the 4 fixed parameters of the initial type-2 SOFLC. Based on the optimization results of SM, a tree structured program formed by the GP will help the SOFLC directly build its best structure for a specific patient according to the patient weight input to the TCI. There are four GP trees to optimize the structure of the IT2 SOFLC corresponding to the FOU width for input type-2 fuzzy sets, two input scaling factors and one output scaling factor respectively. Fig. 5 is an example of the GP tree to define the FOUs for input type-2 fuzzy sets with regards to a patient's weight. The internal nodes of the tree correspond to a function set (F), the variables and constants correspond to terminal set (T). The maximal layer of the GP tree is defined as 17. Using this approach, the FOU of type-2 fuzzy sets becomes flexible to patient specific characteristics as described in Fig. 3. Furthermore, the input and output scaling factors are programs rather than fixed constants which enables the type-2 SOFLC to handle the uncertainties of inter-patient variabilities.

\section{SimULATIONS AND RESULTS}

In this section, our proposed type-2 SM-GP SOFLC is evaluated against variant optimization strategies for tuning the type-2 SOFLC parameters. We also compared our approach with an equivalent type-1 SOFLC based optimized system. To provide a robust comparative evaluation our approach was compared against a GA and SM only optimized SOFLC to evaluate its performance in surgical control simulations. Clinical data on patient characteristics which include age, height and weight were acquired for 20 patients from the National Taiwan University Hospital. These patient parameters were different from those used for optimizing the SOFLCs as described in section IV. Each type of optimized IT2 SOFLC was applied to these 20 patients' characteristics to evaluate their simulated optimization capability and performance. During the simulation, the weight, height and age of the 20 patients were used to calculate metabolic clearance and inter-compartmental clearances which influence propofol concentration in the human body and further leads to differences in BIS output (response).

GA optimization was applied to both type-1 and type-2 SOFLCs. For GA settings, real numbers were adopted for encoding each individual chromosome. The crossover operator used was a Laplace crossover based on Laplace distribution; the mutation operator was power mutation based on power distribution; and the selection technique was tournament selection. Individuals were selected using the genetic crossover and mutation operators for producing the next generation. The maximum number of generations was defined as 200. A population size of 50 was used in order to make the GA more efficient to process more solutions at the same time without increasing the iteration time. Based on the length of chromosome and accuracy requirement, we specified the probability of crossover to be 0.9 and the probability of mutation to be 0.1 to guarantee biodiversity of solutions and avoid the effect of instability or premature convergence.

At the beginning of the surgery, the TCI pump initially provided a bolus to make the patient fall asleep quickly, causing a quick drop of BIS value. The proposed controller began to work after 10 minutes. The entire surgical simulations of 100 minutes were performed emulating realistic surgery durations, during which anesthesia is continually administered and regulated in a patient. In practical clinical settings, EEG readings can be easily influenced by signal noise which make it difficult to produce very precise control. Hence, we simulate the addition of white noise at 5\%,10\% and $20 \%$ between 25 to 30 minutes, 55 to 60 minutes and 85 to 90 minutes of drug administration. Clinically, a BIS value between 40 and 60 is recommended in general anesthesia [33, 52]. In our simulations, the BIS set point was 40 and the duration was 100 minutes representing a realistic timescale for an ear, nose and throat surgical procedure. The steady state error and total propofol dosage were calculated from 10 minutes into each simulation and run up to the end of the simulation. At the same time, the points where the BIS value dipped to 30 were also recorded. This is of vital importance to assess safe anesthesia as BIS values of less than 30 will lead to burst suppression which can be dangerous for patients. Fig. 6-9 show the simulation results for each of the 20 patients to provide a detailed and comprehensive comparison between the different SOFLC optimization strategies applied. Table III shows the average value and standard deviation of steady state error, total dosage and frequency of BIS reaching 30 for the 20 patients sampled.

From Fig. 6 and Fig. 7, it can be concluded that the optimized type-1 SOFLC produce significantly higher errors than the type-2 SOFLC. Some of the simulation results of the type-1 SOFLC failed to reach the set point, which also suggests it is poorly adapting to inter-patient and intra-patient variabilities. The average $M A E_{B I S}$ and $S t d v_{B I S}$ of type-2 SOFLCs are also significantly lower compared with type-1 SOFLC. From Fig. 7 and Fig. 8, it can be observed that SM optimized type-2 SOFLC has less control oscillations than the GA optimized type-2 SOFLC and settles closer to the set point of BIS. This performance is shown to be further enhanced with the SMbased GP optimized SOFLC as observed from Fig. 8 and Fig. 9. The GP optimized type-2 SOFLC reveals greater robustness while handing inter-patient variability since there are only 2 


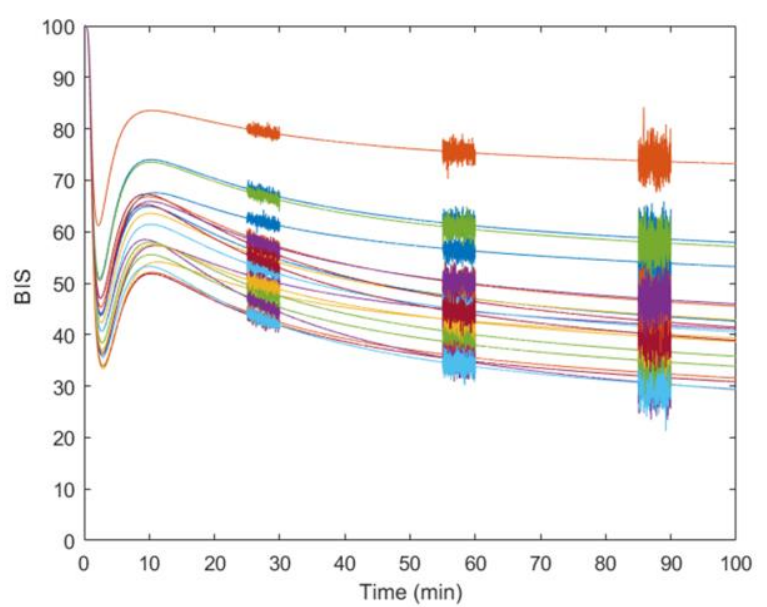

Fig. 6 GA optimized type-1 SOFLC applied to 20 patients

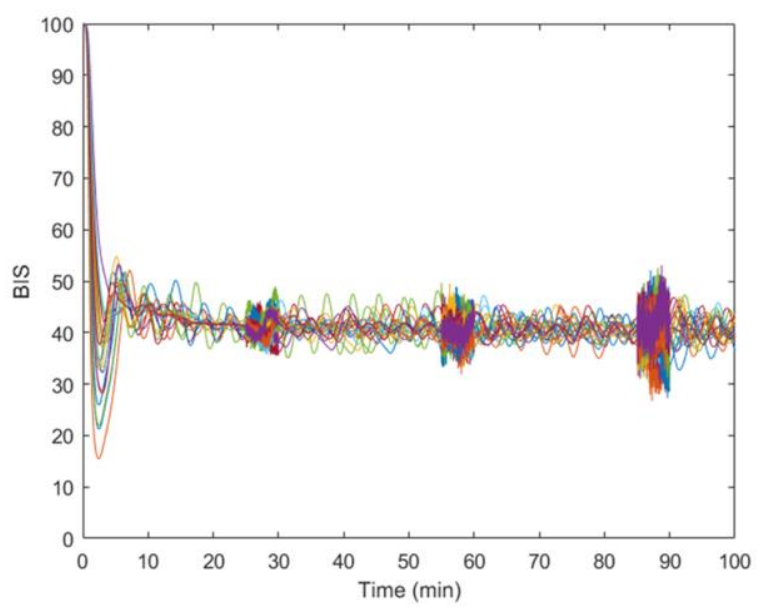

Fig. 8 SM optimized type-2 SOFLC applied to 20 patients

patients whose average $M A E_{B I S}$ are larger than 2 compared to 7 and 5 for the GA and SM respectively. Moreover, one can notice that the average $M A E_{B I S}$ and $S t d v_{B I S}$ produced by SMbased GP optimized SOFLC is 1.4012 and 1.8102, which is significantly lower compared with the SOFLC in previous studies $[10,25]$. The average frequency of BIS falling to below 30 for the GP optimized SOFLC is 0.8 , whereas they are 18.7 and 1.9 for GA and SM optimized SOFLCs respectively. Every time BIS values falls below 30 in the simulation means that the BIS score falls below 30 for 0.01 second in the real clinical procedure. For the proposed GP optimized type-2 SOFLC, as the average frequency of BIS falling to below 30 is 0.8 , the

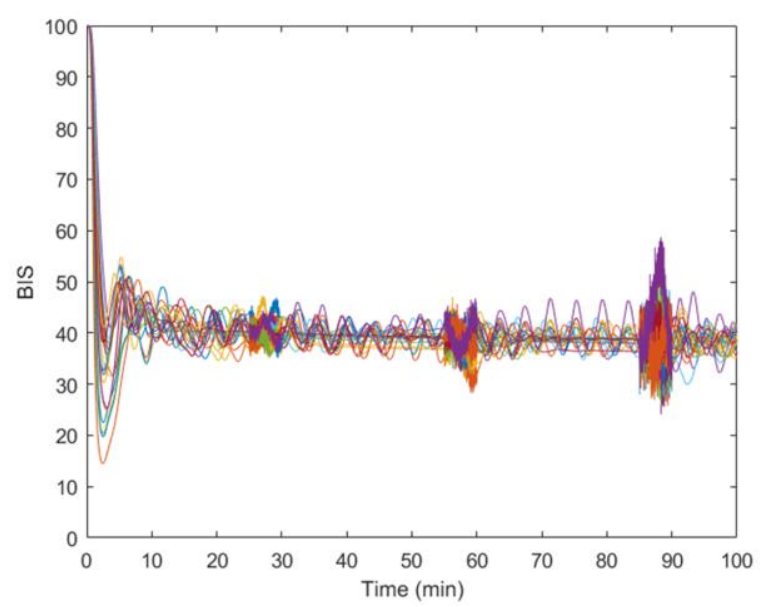

Fig. 7 GA optimized type-2 SOFLC applied to 20 patients

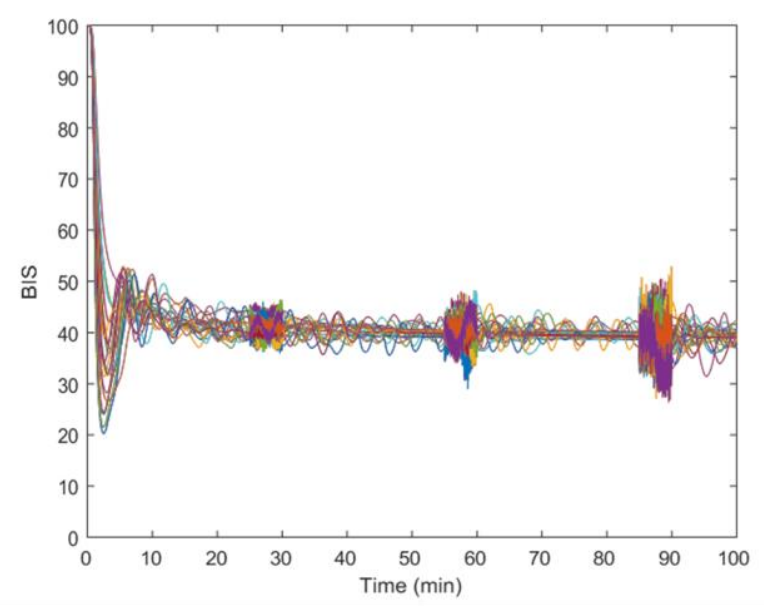

Fig. 9 SM-based GP optimized type-2 SOFLC applied to 20 patients

average actual time duration for BIS dropping below 30 is $0.008 \mathrm{~s}$ throughout the simulation, which is extremely short and considered within acceptable boundaries. The results above show that the SM-based GP optimized SOFLC performs well in dealing with different surgical problems.

A statistical comparison of the controllers was also performed using the Wilcoxon sign-rank tests where significant difference in performance are show as $\mathrm{p}$ values of $<0.05$. The results show that the $\mathrm{p}$ value between the $M A E_{B I S}$ of SM and SM-based GP type-2 systems is 0.002 , proving that the GP combined optimized type-2-SOFLC significantly differs from type-2 SOFLC optimized by SM alone.

TABLE III

THE SIMULATION RESULTS APPLIED TO 20 PATIENTS BY OPTIMIZED SOFLC

\begin{tabular}{|c|c|c|c|c|c|c|c|c|}
\hline \multirow{2}{*}{ Results } & \multicolumn{4}{|c|}{ GA optimized type-1 SOFLC } & \multicolumn{4}{|c|}{ GA optimized type-2 SOFLC } \\
\hline & $M A E_{B I S}$ & $S U M_{\text {prop }}$ & $S t d v_{B I S}$ & $\mathbf{B I S}<\mathbf{3 0}$ & $M A E_{B I S}$ & $S U M_{\text {prop }}$ & $S t d v_{B I S}$ & BIS $<30$ \\
\hline Avg & 10.9799 & 1853.2957 & 5.6885 & 102.9 & 1.8527 & 1874.6966 & 2.3154 & 18.65 \\
\hline Stdv & 8.0243 & 2.7963 & 0.3594 & -- & 0.3301 & 11.8920 & 0.3694 & -- \\
\hline \multirow{2}{*}{ Results } & \multicolumn{4}{|c|}{ SM optimized type-2 SOFLC } & \multicolumn{4}{|c|}{ SM-based GP optimized type-2 SOFLC } \\
\hline & $M A E_{B I S}$ & $S U M_{\text {prop }}$ & $S t d v_{B I S}$ & $\mathrm{BIS}<30$ & $M A E_{B I S}$ & $S U M_{\text {prop }}$ & $S t d v_{B I S}$ & $\mathbf{B I S}<\mathbf{3 0}$ \\
\hline Avg & 1.7891 & 1828.8150 & 1.9996 & 1.90 & 1.4012 & 1845.5976 & 1.8108 & 0.80 \\
\hline Stdv & 0.2976 & 10.9564 & 0.4220 & - & 0.3881 & 17.6070 & 0.2641 & -- \\
\hline
\end{tabular}


The proposed GP optimized type-2 SOFLC is more robust, approximates and more stably maintains the control set point for maintaining BIS in comparison with other accepted approaches for anesthesia control such as the model adaptive controller (MAC) [7, 53] and a linear model predicative controller (LMPC) [54]. In comparison with the general (zSlices) based type-2 SOFLC whose fuzzy sets were defined using data acquired from monitoring physiological parameters of anesthetized patients [10], the optimized interval type-2 SOFLC controller potentially has less processing overheads. The approach however has greater structural flexibility to handle inter-patient variabilities than non-optimized IT2 SOFLCs.

\section{CONCLUSION}

In this paper, different optimization strategies are tested and compared with the simulation of 20 patients, and a novel interval type-2 SOFLC optimized by the combination of SM and GP is proposed. Since the width of FOU, input and output scaling factors are programs rather than constants, this alternative strategy for the optimization of type-2 SOFLC has been shown to be effective in producing improved robustness under different circumstance with varying noise based on a comparison with GA optimized type-2 and type-1 SOFLC to regulate BIS during general anesthesia. The inter-patient variability is also shown to be handled well by the proposed optimization strategy.

A limitation of our proposed GP optimization strategy is that it is time-consuming if more parameters are included in the optimization domain since it is a single input and single output regression system. Hence there is a need for four GP trees for each of the four system parameters respectively. So, the GP optimization process needs to run four times to build each GP tree. General type-2 fuzzy sets can be considered in the future as they provide higher design dimensions for modeling uncertainties. However, the increased numbers of parameters in the MF will lead to a more complicated solution space for the GP resulting in a more time-consuming training process.

This problem can however be solved if we adopt Cartesian Genetic Programming (CGP) [55], which is a multi-input and multi-output optimization algorithm representing computational structures as a string of integers. Applying CGP, the optimization strategy can more efficiently involve multiple parameters as the GP optimization process only needs to be run once for all system parameters. More patient information including age and height can be used to define the controller structure as compared to currently only considering the weight of patient to generate type-2 fuzzy sets and scaling factors. Furthermore, parameters inside the SO mechanism can be included in the optimization procedure using the multi-input and multi-output algorithm which might be a solution for reducing oscillation of SOFLC.

Future work will investigate the use of more patient information and SOFLC parameters represented using general type-2 fuzzy sets that could be optimized using a CGP.

\section{REFERENCES}

[1] Levin Kuhlmann, Jonathan H. Manton, FTracking, "Electroencephalographic Changes Using Distributions of Linear Models: Application to Propofol Based Depth of Anesthesia Monitoring," IEEE Trans. Biomed. Eng., 64(4):870-881, Apr. 2017.

[2] K Leslie, O Clavisi, J Hargrove, "Target-controlled infusion versus manually-controlled infusion of propofol for general anaesthesia or sedation in adults," Anesthesia and analgesia., 107(6):2089, Dec. 2008.

[3] C.H. Ting, R.H. Arnott, D.A. Linkens, A. Angel, "Migrating from target-controlled infusion to closed-loop control in general anaesthesia," Computer Methods and Programs in Biomedicine., 75(2), 127-139, 2004.

[4] Pajic Miroslav et al., "Model-Driven Safety Analysis of Closed-Loop Medical Systems," IEEE Trans. Industrial Informatics., 10(1):3-16, Feb. 2014.

[5] Klaske van HeusdenGuy A. Dumont et al., "Design and clinical evaluation of robust PID control of propofol anesthesia in children," IEEE Trans. Control Systems Technology., 22(2):491-501, Mar. 2014.

[6] Liu N., Chazot T., Genty A. et al., "Titration of propofol for anesthetic induction and maintenance guided by the bispectral index: closed-loop versus manual control: a prospective, randomized, multicenter study," The Journal of the American Society of Anesthesiologists., 104(4), 686-695, 2006.

[7] Ionescu, C.M, De Keyser, R. et al., "Robust Predictive Control Strategy Applied for Propofol Dosing Using BIS as a Controlled Variable During Anesthesia," IEEE Trans. Biomedical Engineering., 55(9):2161-2170, Sep. 2008.

[8] M. F. Abbod, D. G. von Keyserlingk, D. A. Linkens, M. Mahfouf, "Survey of utilisation of fuzzy technology in medicine and healthcare," Fuzzy Sets and Systems., vol. 120, pp. 331-349, 2001.

[9] A. O. Esogbue and R. C. Elder, "Measurement and valuation of a fuzzy mathematical model for medical diagnosis," Fuzzy Sets and Systems., vol. 10, pp. 223-242, 1983.

[10] F. Doctor, C.-H. Syue, Y.-X. Liu, J.-S. Shieh, R. Iqbal, "Type-2 fuzzy sets applied to multivariable self-organizing fuzzy logic controllers for regulating anesthesia," Applied Soft Computing., vol. 38, pp. 872-889, 2016.

[11] John, Robert and Simon Coupland. "Type-2 fuzzy logic: challenges and misconceptions [discussion forum]." IEEE Computational Intelligence Magazine., 7.3: 48-52, 2012.

[12] Ramirez, Eduardo, Patricia Melin, and German PradoArechiga. "Hybrid model based on neural networks, type-1 and type-2 fuzzy systems for 2-lead cardiac arrhythmia classification." Expert Systems with Applications., 126: 295307, 2019.

[13] Guzmán, Juan Carlos et al., "Optimal genetic design of type1 and interval type-2 fuzzy systems for blood pressure level classification." Axioms., 8(1): 8, 2019.

[14] Ontiveros-Robles, Emanuel, Patricia Melin, and Oscar Castillo. "Comparative analysis of noise robustness of type 2 fuzzy logic controllers." Kybernetika., 54(1): 175-201, 2018.

[15] Castillo, Oscar, et al. "A generalized type-2 fuzzy granular approach with applications to aerospace." Information Sciences., 354: 165-177, 2016.

[16] Castillo, Oscar et al., "A comparative study of type-1 fuzzy logic systems, interval type-2 fuzzy logic systems and generalized type-2 fuzzy logic systems in control problems." Information Sciences., 354: 257-274, 2016.

[17] Cervantes, Leticia, and Oscar Castillo. "Type-2 fuzzy logic aggregation of multiple fuzzy controllers for airplane flight control." Information Sciences., 324: 247-256, 2015.

[18] Procyk, Tom J., and Ebrahim H. Mamdani. "A linguistic selforganizing process controller." Automatica., 15.1: 15-30, 1979.

[19] Liu, Yan-Xin et al., "Performance analysis of extracted rulebase multivariable type-2 self-organizing fuzzy logic controller applied to anesthesia." BioMed research international 2014., 2014. 
[20] El-Bardini, Mohammad, and Ahmad M. El-Nagar. "Direct adaptive interval type-2 fuzzy logic controller for the multivariable anesthesia system." Ain Shams Engineering Journal., 2.3-4: 149-160, 2011.

[21] El-Nagar, Ahmad M., and Mohammad El-Bardini. "Interval type-2 fuzzy neural network controller for a multivariable anesthesia system based on a hardware-in-the-loop simulation." Artificial intelligence in medicine., 61.1: 1-10, 2014.

[22] Taheriyan, Fatemeh, Mandana sadat Ghafourian, and Amin Noori. "Applying GA Optimization Algorithm for Interval Type-2 Fuzzy Logic Controller Parameters of Multivariable Anesthesia System." Electrical Engineering (ICEE), Iranian Conference on. IEEE, 2018.

[23] Méndez, Juan A. et al., "Adaptive fuzzy predictive controller for anesthesia delivery." Control Engineering Practice., 46: $1-9,2016$

[24] El-Nagar, Ahmad M., and Mohammad El-Bardini. "Parallel realization for self-tuning interval type-2 fuzzy controller." Engineering Applications of Artificial Intelligence., 61: 820, 2017.

[25] Y.-X. Liu, J.-S. Shieh, S.-Z. Fan, F. Doctor, and K.-K. Jen, "Genetic type-2 self-organising fuzzy logic controller applied to anaesthesia," in 2015 Conference on Technologies and Applications of Artificial Intelligence (TAAI), Taiwan, 2015, pp. 83-88.

[26] Alexander I.J. Forrester, Andy J.Keane, "Recent advances in surrogate-based optimization," Progress in aerospace sciences., 45.1-3: 50-79, 2009.

[27] Samui, Pijush, Chakraborty, Subrata, Kim, Dookie, "Fuzzy Structural Analysis Using Surrogate Models," Modeling and Simulation Techniques in Structural Engineering. IGI Global, pp. 239-265, 2017.

[28] Vladislavleva, Ekaterina J., Guido F. Smits, and Dick Den Hertog. "Order of nonlinearity as a complexity measure for models generated by symbolic regression via pareto genetic programming." IEEE Transactions on Evolutionary Computation., 13.2: 333-349, 2008.

[29] Stijven, Sean et al., "Prime-Time: Symbolic Regression Takes Its Place in the Real World." in Genetic Programming Theory and Practice XIII. Springer, Cham, 2016, pp. 241260.

[30] Poli, Riccardo, et al. "A field guide to genetic programming," Lulu. com, 2008. [Online]. Available: http://lulu.com, 2008.

[31] Tunstel, Edward, and Mo Jamshidi., "On genetic programming of fuzzy rule-based systems for intelligent control." Intelligent Automation \& Soft Computing., 2.3: 271-284, 1996.

[32] Alba, Enrique, Carlos Cotta, and José $\mathrm{M}^{\mathrm{a}}$ Troya. "Evolutionary design of fuzzy logic controllers using strongly-typed GP." Mathware and Soft Computing., 6.1: 109-124, 1999.

[33] I. J. Rampil, "A primer for EEG signal processing in anesthesia," The Journal of the American Society of Anesthesiologists., vol. 89, pp. 980-1002, 1998.

[34] T. W. Schnider et al., "The influence of method of administration and covariates on the pharmacokinetics of propofol in adult volunteers," Anesthesiologists., vol. 88, pp. 1170-1182, 1998.

[35] Chakravarty Sourish et al., "Pharmacodynamic modeling of propofol-induced general anesthesia in young adults," 2017 IEEE Healthcare Innovation Point-of-Care Technologies (HI-POCT), pp. 44-47, Nov. 2017.

[36] J. M. Mendel, "Type-2 fuzzy sets and systems: an overview," IEEE computational intelligence magazine., vol. 2, pp. 2029, 2007.

[37] J. M. Mendel, Uncertain Rule-Based Fuzzy Logic Systems: Introduction and New Directions, Upper Saddle River, New Jersey: Prentice Hall, 2001.

[38] J. M. Mendel and R. B. John, "Type-2 fuzzy sets made simple," IEEE Trans. Fuzzy Systems., vol. 10, pp. 117-127, 2002.

[39] Y.-X. Liu, F. Doctor, S.-Z. Fan, and J.-S. Shieh, "Performance Analysis of Extracted Rule-Base Multivariable Type-2 Self-Organizing Fuzzy Logic
Controller Applied to Anesthesia," BioMed Research International, vol. 2014, pp. 19, 2014.

[40] J. Shieh, M. Abbod, C. Hsu, S. Huang, Y. Han, and S. Fan, "Monitoring and control of anesthesia using multivariable self-organizing fuzzy logic structure," Fuzzy Systems in Bioinformatics and Computational Biology, pp. 273-295: Springer, 2009.

[41] C.-T. Chuang, S.-Z. Fan, and J.-S. Shieh, "Rule extraction by fuzzy modeling algorithm for fuzzy logic control of cisatracurium as a neuromuscular block," Engineering Applications of Artificial Intelligence., vol. 22, no. 1, pp. 129-140, 2009.

[42] N. N. Karnik, and J. M. Mendel, "Centroid of a type-2 fuzzy set," Information Sciences., vol. 132, no. 1, pp. 195-220, 2001.

[43] Q. Liang, and J. M. Mendel, "Interval type-2 fuzzy logic systems: theory and design," IEEE Trans. Fuzzy Systems., vol. 8, no. 5, pp. 535-550, 2000.

[44] Karnik, Nilesh N., and Jerry M. Mendel. "Type-2 fuzzy logic systems: type-reduction." in 1998 IEEE International Conference on Systems, Man, and Cybernetics, (Cat. No. 98CH36218), 1998, Vol. 2.

[45] D. Wu, and M. Nie, "Comparison and practical implementation of type-reduction algorithms for type-2 fuzzy sets and systems." in 2011 IEEE International Conference on Fuzzy Systems (FUZZ-IEEE 2011), 2011, pp. 2131-2138.

[46] Tong, R.M, "Synthesis of fuzzy models for industrial processes - some recent results." International Journal of General Systems 4, 143-162, 1978.

[47] D. A. Linkens, and S. Hasnain, "Self-organising fuzzy logic control and application to muscle relaxant anaesthesia." IEE Proceedings D (Control Theory and Applications). Vol. 138. No. 3. IET Digital Library, 1991.

[48] J. Mu"ller, C.A. Shoemaker, and R. Pich'e. SO-MI, "A surrogate model algorithm for computationally expensive nonlinear mixed-integer black-box global optimization problems. Computers and Operations Research," Computers and Operations Research., 40(5):1383-1400, May. 2013.

[49] Luna, J.M. Pechenizkiy, M.del Jesus, M.J. Ventura, S. "Mining Context-Aware Association Rules Using GrammarBased Genetic Programming, IEEE Trans. Cybern. Cybernetics., 48(11):3030-3044, Nov. 2018.

[50] Austel, Vernon, et al. "Globally optimal symbolic regression." arXiv preprint arXiv:1710.10720 (2017).

[51] Smits, Guido F., and Mark Kotanchek. "Pareto-front exploitation in symbolic regression." in Genetic programming theory and practice II. Springer, Boston, MA, 2005, pp. 283-299.

[52] J.-Y.Lan, M. F. Abbod, R.-G. Yeh, S.-Z. Fan, and J.-S. Shieh, "Review: intelligent modeling and control in anesthesia," Journal of Medical and Biological Engineering., vol. 32, no. 5, pp. 293-307, 2012.

[53] Sawaguchi Y, Furutani E, Shirakami G, Araki M, Fukuda K, "A model-predictive hypnosis control system under total intravenous anesthesia," IEEE Trans. Biomed Eng., 55(3):874-87, Mar. 2008.

[54] Zhusubaliyev, Zhanybai T., Alexander Medvedev, and Margarida M. Silva. "Bifurcation analysis of PID-controlled neuromuscular blockade in closed-loop anesthesia." Journal of Process Control., 25: 152-163, 2015.

[55] Miller, Julian F. "Cartesian genetic programming." In Proceedings of the 10th annual conference companion on Genetic and evolutionary computation, ACM, 2008, pp. 2701-2726, 


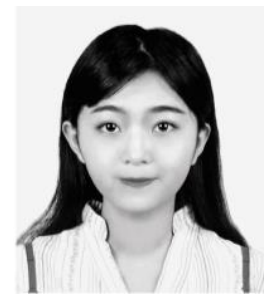

Zi-Xiao Wei was born in Nanjing, Jiangsu, China in 1997. She received her B.Sc. degree in mechanical engineering from Yuan Ze University, Chungli, Taiwan, in 2019. She is currently pursuing her M.Sc. degree in the Department of Mechanical Engineering at the National University of Singapore. Her research interests include 3D printing, evolutionary algorithms, and intelligent systems.

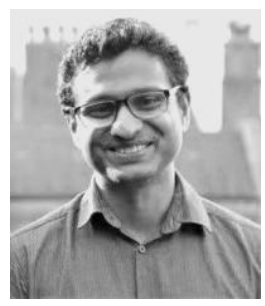

Faiyaz Doctor (M'08) received the B.Sc. degree in computer science and artificial intelligence from University of Birmingham in 1998. M.Sc. degree in artificial intelligent agents in 2001 and $\mathrm{PhD}$. degree in computer science in 2006 from the University of Essex, U. K. He is currently a Senior Lecturer with the School of Computer Science and Electronic Engineering, head of the Intelligent Connected Societies Group and member of the Centre for Computational Intelligence and Intelligent Embedded Systems and Environments Group at the University of Essex.

He has over 15 years' experience in the design and implementation of intelligence systems for real world applications with projects funded by the Royal Academy of Engineers, Innovate UK, Harvard University, and the Newton Fund. His research interests are in computational intelligence emphasising on fuzzy systems, deep learning, evolutionary algorithms, hybrid machine learning techniques, ambient intelligence and pervasive computing.

Dr Doctor has published over 75 papers in peer-reviewed international journals, conferences, and workshops and is a member of the IEEE and IEEE Computational Intelligence Society.

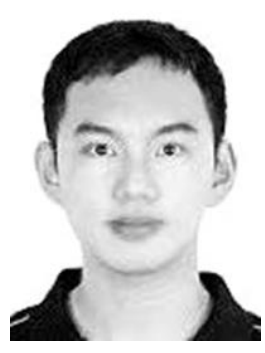

Yan-Xin Liu received his B.Sc. degree in mechanical engineering from Yuan $\mathrm{Ze}$ University, Chungli, Taiwan, in 2016 and received his M.Sc. degree in electrical engineering from the Technical University of Denmark, Kongens Lyngby, Denmark, in 2018. He is currently pursuing his Ph.D. degree in the Department of Physics at the Technical University of Denmark. His research interests include microelectromechanical systems, optomechanics, and intelligent systems.

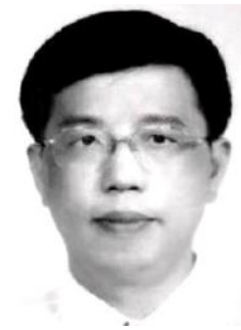

Shou-Zen Fan received the Ph.D. degree from the National Taiwan University in 1994. He is currently an Associate Professor with National Taiwan University and the Director of General Anesthesiology with the National Taiwan University Hospital. His research interests include pediatric anesthesia and pain management, airway management, anesthesia for liver transplantation, and anesthetic automatic control and expert systems.

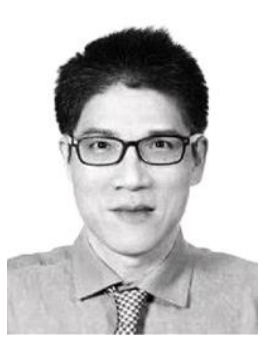

Jiann-Shing Shieh received the B.S. and M.S. degrees in chemical engineering from National Cheng Kung University, Taiwan, in 1983 and 1986, respectively, and the $\mathrm{Ph} . \mathrm{D}$. degree in automatic control and systems engineering from the University of Sheffield, U.K., in 1995. He is currently a Professor with the Department of Mechanical Engineering, also a Joint Professor with the Graduate School of Biotechnology and Bioengineering, and also serves as the Dean of the College of Engineering at Yuan Ze University, Taiwan. His research interests are focused on biomedical engineering, particularly in bio-signal processing of ECG, BP, EEG, SPO2, center of pressure (COP) position signals, artificial intelligent analysis and control, medical automation, pain model and control, critical care medicine monitoring and control, dynamic cerebral autoregulation research, and brain death index research.

Professor Shieh has published over 130 papers in peerreviewed international journals. He also serves a Section Editor of Journal of Clinical Medicine and an Academic Editor of Journal of Healthcare Engineering. 